\title{
Pain Intensity And Attribution Mediate The Impact Of Patient Weight And Gender On Activity Recommendations For Chronic Pain
}

\author{
This article was published in the following Dove Press journal: \\ Journal of Pain Research
}

\author{
Lauren E Mehok' \\ Megan M Miller' \\ Zina Trost ${ }^{2}$ \\ Liesbet Goubert $\left(\mathbb{D}^{3}\right.$ \\ Lies De Ruddere ${ }^{3}$ \\ Adam T Hirsh' \\ 'Department of Psychology, Indiana \\ University - Purdue University \\ Indianapolis, Indianapolis, IN, USA; \\ ${ }^{2}$ Department of Psychology, University of \\ Alabama at Birmingham, Birmingham, AL, \\ USA; ${ }^{3}$ Department of Experimental- \\ Clinical and Health Psychology, Ghent \\ University, Ghent, Belgium
}

Correspondence: Adam T Hirsh

Department of Psychology, Indiana

University - Purdue University

Indianapolis, LD 124, 402 N. Blackford St.,

Indianapolis, IN 46202, USA

Tel + I 3172746942

Fax +I 3172746756

Email athirsh@iupui.edu
Background and purpose: Despite the notable benefits of physical activity for chronic pain, a large proportion of patients with chronic pain report that they do not receive activityrelated recommendations from their providers. Research suggests that patient factors such as weight and gender influence activity-related recommendations for chronic pain. Research also suggests that appraisals of the intensity and cause of pain may explain these weight and gender effects. We investigated the influence of patient weight and gender on observers' likelihood of recommending activity-related treatments for pain. We also explored the mediating effects of observers' ratings of pain severity and the extent to which pain was due to medical and lifestyle factors (pain attribution).

Patients and methods: Healthy young adults ( $\mathrm{N}=616 ; 76 \%$ female) viewed videos (Ghent Pain Videos of Daily Activities) and vignettes of 4 patients with chronic back pain performing a standardized functional task. Patients varied by gender (female, male) and weight (normal, obese), but were otherwise equivalent on demographic characteristics and pain behaviors. Participants rated how much pain they perceived the patients to be experiencing, the extent to which they attributed the pain to medical and lifestyle factors, and their likelihood of recommending exercise, physical therapy (PT), and rest.

Results: Patient weight and gender significantly interacted to influence exercise, PT, and rest recommendations. Both pain intensity and pain attribution mediated the relationships between patient weight and activity recommendations; however, these mediation effects differed across gender and recommendation type.

Conclusion: Patient weight and gender influenced laypeople's activity recommendations for chronic pain. Moreover, the results suggest that observers' perceptions of pain intensity and pain attributions are mechanisms underlying these effects. If these findings are replicated in providers, interventions may need to be developed to reduce provider biases and increase their recognition of the benefits of physical activity for chronic pain.

Keywords: pain, weight, gender, physical activity, recommendations

\section{Introduction}

Physical activity, including exercise and physical therapy (PT), is a critical component of chronic pain care. Exercise has been found to reduce pain, ${ }^{1-4}$ prevent and reduce obesity, ${ }^{1,5,6}$ and increase quality of life ${ }^{1}$ in patients with chronic pain. Early PT following a new primary care consultation for low back pain has been associated with lower medical costs and healthcare utilization. ${ }^{7}$ Despite these benefits, many patients with chronic pain do not receive activity-related recommendations from their providers. Some findings indicate that less than half of patients with 
arthritis, back pain, and neck pain are advised to be physically active. ${ }^{7,8}$ Furthermore, restrictive recommendations such as bed-rest are common, ${ }^{9}$ despite such behaviors being discouraged by clinical guidelines for pain. ${ }^{2}$

Several patient factors have been shown to influence providers' treatment decisions, both in general ${ }^{10}$ and specifically in regard to chronic pain. ${ }^{11-15}$ Weight is one such factor. Chronic pain and obesity are highly comorbid, with bidirectional adverse impacts. ${ }^{16-20}$ Moreover, laypersons hold biases about people with obesity, ${ }^{21}$ including biases about physical activity. ${ }^{22,23}$ For example, strong anti-fat biases have been found in gym goers and fitness professionals. $^{24}$ Negative attitudes and stereotypes about patients with overweight/obesity have also been identified in medical students and physicians, ${ }^{25}$ where patients' weight has been found to influence provider decisions. Specifically, providers spend less time with patients with overweight/obesity and may fail to consider treatment options beyond advising patients to lose weight. ${ }^{26}$ Alternatively, some evidence suggests that patients with chronic pain and overweight/obesity are actually more likely than normal weight patients to receive activity advice from their doctor. ${ }^{14}$ Gender is another patient factor found to influence physical activity recommendations. ${ }^{14}$ In the general public, gender stereotypes related to physical activity suggest that men are more athletic than women and that men and women participate in different types of physical activity. ${ }^{27,28}$ Like weight, patient gender has been shown to influence providers' treatment recommendations for pain. ${ }^{29}$ Women are at greater risk of having their pain undertreated compared to men. For example, women in pain are more likely to have their symptoms attributed to psychological factors such as anxiety and less likely to be prescribed/administered analgesic medication. ${ }^{29-31}$ On the other hand, one population-based study found that women with arthritis-related pain were more likely to report having received activity advice from their doctor. ${ }^{14}$

In addition to their independent impact on pain-related decisions, emerging evidence suggests patient weight and gender have interactive effects. For example, laypersons, medical students, and physicians have been found to give different treatment recommendations to men and women across weight categories. ${ }^{15,25}$ In a study on treatment recommendations made by healthy young adults, Miller et al found that male patients of normal weight were more likely to be recommended opioids than female patients, but the pattern was reversed if they had obesity. ${ }^{15}$ Previous literature also suggests that observer appraisals regarding the source and intensity of patient pain are likewise affected by patient weight and gender. In terms of pain intensity, a large body of work indicates that pain is more likely to be underestimated and discounted in women than men. ${ }^{15,29,32-34}$ This underestimation may be due to the fact that women are more pain sensitive and are also more likely to have their pain attributed to psychological factors. ${ }^{35}$ A recent study found that pain intensity ratings were different depending on both the weight and gender of the patient. ${ }^{15}$ Pain attribution, or the believed cause of pain, is another appraisal that appears to differ depending on the weight and gender of the patient. ${ }^{15,29}$ Miller et $\mathrm{al}^{15}$ found that laypersons rated medical factors as the more likely cause of pain for male patients with normal and overweight compared to weight-matched females, whereas female patients of all weight categories were more likely to have the cause of their pain attributed to psychological factors compared to weight-matched male patients. Collectively, these results suggest that weight and gender differences in activity-related recommendations for pain may be driven by differences in observers' (laypeople and providers) perception regarding the intensity and source of pain in men vs women and normal vs overweight patients.

The purpose of this study was to: 1) examine how patient weight and gender influence layperson's activityrelated recommendations for chronic pain, and 2) explore the role of pain intensity and pain attribution in this context. We hypothesized that patient weight and gender would have unique and interactive effects on participants' likelihood of recommending exercise, PT, and rest. Additionally, we hypothesized that participants' perceptions and attributions of patients' pain would mediate (ie, explain) the relationship between weight and activityrelated recommendations, and that these mediation effects would differ for male and female patients. This study aims to contribute important new information about weight and gender biases in laypeople, which will advance our understanding of the extent to which such biases are products of society at large or specific to the healthcare setting. Such understanding is key to intervention development; thus, these findings will be of interest to clinicians working with patients with chronic pain and educators training the next generation of clinical providers. The clinical relevance of this study is also heightened by the fact that chronic pain and obesity are public health concerns on their own, are highly comorbid, and adversely impact each other. $^{16-20}$ 


\section{Methods}

\section{Participants}

This study is a secondary analysis of data used in a previous paper. ${ }^{15}$ Six hundred and sixteen undergraduates were recruited from the University of North Texas (UNT, $\mathrm{n}=189 ; 69.3 \%$ female) and Indiana University - Purdue University Indianapolis (IUPUI, $\mathrm{n}=427 ; 78.9 \%$ female). Participants were recruited through an online research participation platform and were compensated with course credit.

\section{Procedure}

All procedures were completed online and approved by the IUPUI and UNT institutional review boards. Participants used a unique ID code to access an online portal. After providing informed consent and demographic information, participants were given the following directions:

You will now see pictures of six different chronic pain patients. Imagine that these patients are seeking treatment from you. Along with each picture, you will get specific information about each patient. Following the picture of each patient, you will see a video of the patient performing a physical activity as part of their standard physical evaluation. In total, you will be presented with six video clips and will be asked questions following each video. Closely examine all of the information for each patient. This study aims to understand how laypeople formulate an impression of patients.

Patient videos and vignettes were then displayed in random order, and participants made recommendation ratings and appraisals for each patient-vignette pair. The study took approximately 45 mins to complete.

\section{Stimulus Set}

Participants viewed videos of patients with chronic low back pain who consented to be videotaped for research purposes. Eight videos that varied by gender (male or female) and weight (underweight, normal, overweight, or obese) were selected from the Ghent Pain Videos of Daily Activities (G-PAVIDA) ${ }^{12}$ for the parent study. Readers are referred elsewhere ${ }^{12,15}$ for detailed descriptions of the procedures used to generate these videos, as well as the process of categorizing and matching videos across patient demographic and clinical variables. Readers may also contact Dr Liesbet Goubert (Liesbet.Goubert@UGent.be) for more information on G-PAVIDA and their use for research purposes. Briefly, all patients were Caucasian adults (mean age $=52.4[12.3]$ years) who were matched on attractiveness across weight categories. Body mass index (BMI) standards established by the Centers for Disease Control and Prevention ${ }^{36}$ were used to categorize patients into underweight, normal weight, overweight, or obese categories. To confirm the subjective salience of these objective weight classifications, we conducted an unpublished pilot study wherein healthy young adults categorized (twice for reliability purposes) the patients into underweight, normal weight, overweight, and obese categories. These subjective ratings were then compared to the objective BMI data to select the patients that best represented their respective weight categories. Each video included a full-length frontal view of a patient performing a standardized sit-to-stand task. Pain expression/behavior was matched at a moderate level for each patient. We categorized and matched the aforementioned patient variables across videos so as to mitigate potential confounding effects, thus, resulting in stronger inferences about the effects of patient weight and gender on participants' ratings. Each video was accompanied by a text vignette that included relevant personal (ie, occupation) and medical (ie, details of pain) information; these vignettes were randomly paired with videos of gender-concordant patients (see supplementary materials). Based on the aims of this paper, we analyzed the four videos that focused on patients who were normal weight or had obesity and excluded the videos of patients in underweight and overweight categories.

\section{Measures}

After viewing each patient, participants made several appraisal ratings and indicated how likely they were to make various activity-related recommendations. Participants used separate digital VASs ranging from 0 to 100 to make their ratings.

\section{Patient Appraisals}

Pain intensity was assessed using the following question: "How much pain do you think this patient was experiencing?" [No pain to Worst possible pain].

Pain attribution was assessed using two questions: "In your opinion, what proportion of the patient's pain is likely due to lifestyle factors (eg, diet, exercise)?" [0\% to $100 \%$ ] and "In your opinion, what proportion of the patient's pain is likely due to medical factors (eg, nerve or muscle damage)?" [0\% to $100 \%$ ]. To create a single pain attribution variable, a difference score was created for each participant by subtracting their VAS rating for lifestyle 
factors from their VAS rating for medical factors. Thus, each participant had a single continuous rating of attribution, such that higher ratings indicated that participants perceived the patients' pain was more due to medical factors than lifestyle factors.

\section{Treatment Recommendations}

Participants made the following recommendations for each patient: "How likely are you to recommend that this patient:" 1) "Engage in lifestyle activities such as diet and exercise?" [Not at all likely to Extremely likely], 2) "Seek consultation from a physical therapist?" [Not at all likely to Extremely likely], and 3) "Rest; greatly reduce their physical activity?" [Not at all likely to Extremely likely].

\section{Data Analytic Approach}

SPSS version 24 was used for all analyses.

Descriptive statistics were used to summarize participant characteristics. Visual inspection of the scatterplots indicated that there were no outliers, and this was confirmed by the fact that all values were within 3.29 standard deviations of the mean. ${ }^{37}$ Thus, all data were retained for analyses. Separate $2 \times 2$ repeated measures analyses of variance were used to examine the effect of patient weight (normal or obese) and patient gender (male or female) on participants' recommendation ratings for exercise, PT, and rest. Main and interaction effects were tested for each analysis. If the interaction effects were significant, the main effects were not reported.

Repeated measures parallel mediation analyses were conducted using Montoya and Hayes' MEMORE macro ${ }^{12}$ with 5000 bootstrap samples to examine the extent to which participants' appraisals (ie, pain intensity and attribution [difference score]) mediated the relationship between patient weight/gender and participants' treatment recommendations. Separate parallel mediation models were conducted for male and female patients. These mediation analyses allowed us to examine possible mechanisms (ie, pain intensity and attribution) explaining the relationship between patient weight and treatment recommendations.

\section{Results}

The final sample was $65.6 \%$ White, 9.7\% Black, 9.4\% Asian, 6.5\% Multi-racial, 5.5\% Hispanic, 0.8\% Alaskan Native/Native American, $0.3 \%$ Native Hawaiian or Pacific Islander, and $2.2 \%$ not specified. The sample was $76 \%$ female and had a mean age of 20.26 years $(\mathrm{SD}=4.53)$.

\section{Weight $\times$ Gender Interactions}

Exercise Recommendations

The results indicated a significant interaction between patients' weight and gender $(F(1,614)=22.87, \mathrm{p}<0.001$, $\eta_{\mathrm{p}}{ }^{2}=0.04$ ) for exercise recommendations (see Figure 1). Patients with obesity received significantly higher exercise recommendations than their gender-matched normal weight counterparts, and this effect was more pronounced for female patients (mean difference [MD] $=20.89, \mathrm{p}<0.001)$ than male patients $(\mathrm{MD}=14.25$, $\mathrm{p}<0.001)$.

\section{PT Recommendations}

The results indicated a significant interaction between patients' weight and gender $(F(1,614)=121.72, \mathrm{p}<0.001$, $\eta_{\mathrm{p}}{ }^{2}=0.17$ ) for PT recommendations (see Figure 1). Compared to normal weight women, women with obesity received significantly higher PT recommendations $(\mathrm{MD}=12.24, \mathrm{p}<0.001)$, but this pattern was reversed for normal weight and men with obesity $(\mathrm{MD}=-7.17, \mathrm{p}<0.001)$.

\section{Rest Recommendations}

The results indicated a significant interaction between patients' weight and gender $(F(1,614)=31.35, \mathrm{p}<0.001$, $\left.\eta_{\mathrm{p}}{ }^{2}=0.05\right)$ for recommendations to rest (see Figure 1). Normal weight men were significantly more likely to receive a rest recommendation than were men with obesity $(\mathrm{MD}=11.10, \mathrm{p}<0.001)$. Although a similar pattern emerged for normal weight women and women with obesity, the difference was not significant $(\mathrm{MD}=2.20$, $\mathrm{p}=0.086$ ).

\section{Mediation Analyses}

Exercise Recommendations

For male patients, there was a significant total indirect effect of patient weight on the likelihood of receiving an exercise recommendation via pain intensity and pain attribution (total indirect effect $=-5.88$; 95\% CI: -7.90 , -4.02 ). However, only pain attribution ratings (indirect effect $=-5.72$; 95\% CI: $-7.84,-3.84)$, but not pain intensity ratings (indirect effect $=-0.16$; 95\% CI: $-1.05,0.70$ ), significantly mediated the effect of patient weight on participants' exercise recommendations. Compared to their normal weight counterparts, patients with obesity were more likely to have their pain attributed to lifestyle factors than to medical factors, which was associated with a higher likelihood of recommending exercise for male patients. Pairwise contrasts indicated that the 


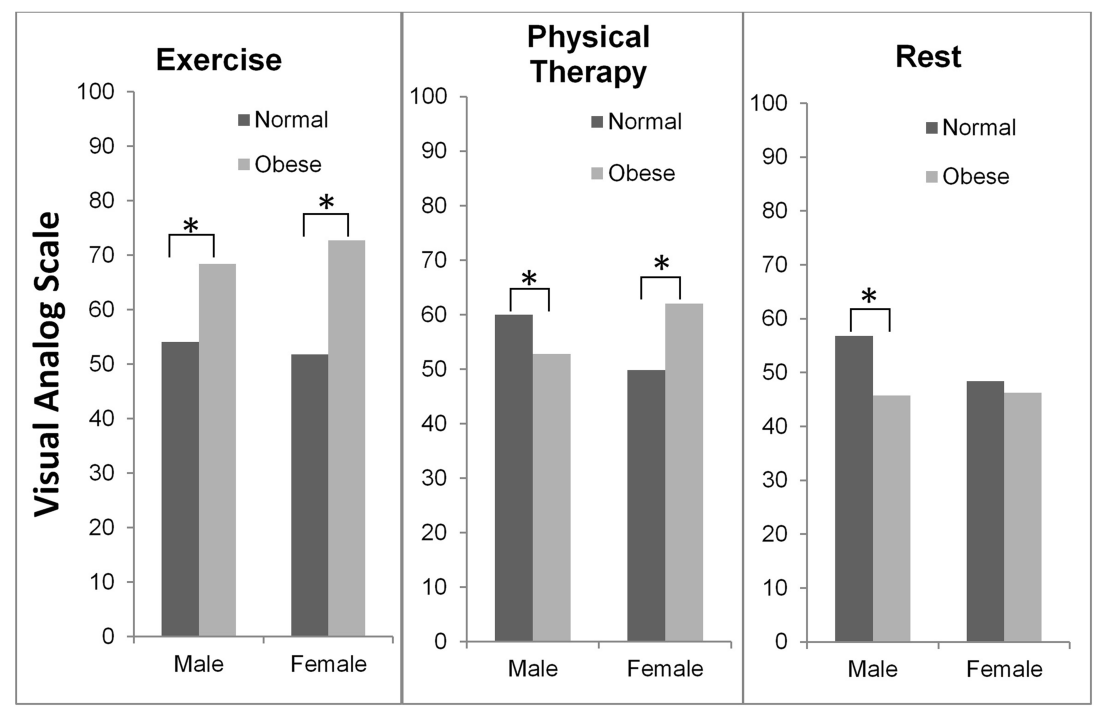

Figure I Rated likelihood of giving a physical activity recommendation.

Note: There was a significant interaction between patient weight and gender on participants' exercise, physical therapy, and rest recommendations. *P<0.05 for the mean differences.

indirect path through pain attribution was significantly larger than the indirect path through pain intensity (contrast $=5.56$; 95\% CI: 3.29, 8.09).

For female patients, there was a significant total indirect effect of patient weight on the likelihood of receiving an exercise recommendation via pain intensity and pain attribution (total indirect effect $=-10.36$; 95\% CI: $-14.35,-6.43)$. Both pain intensity (indirect effect= $-4.42 ; 95 \% \mathrm{CI}:-7.58,-1.42)$ and pain attribution (indirect effect $=-5.94 ; 95 \% \mathrm{CI}:-8.21,-3.76$ ) ratings significantly mediated the effect of patient weight on exercise recommendations. Compared to their normal weight counterparts, female patients with obesity were perceived to be in more pain and were more likely to have their pain attributed to lifestyle factors than to medical factors, and these differences were associated with a higher likelihood of female patients receiving an exercise recommendation. Pairwise contrasts indicated that the indirect paths through pain intensity and pain attribution were not significantly different (contrast $=1.12 ; 95 \%$ CI: $-2.19,5.10$ ). Figure 2 shows the results of the analysis in the model.

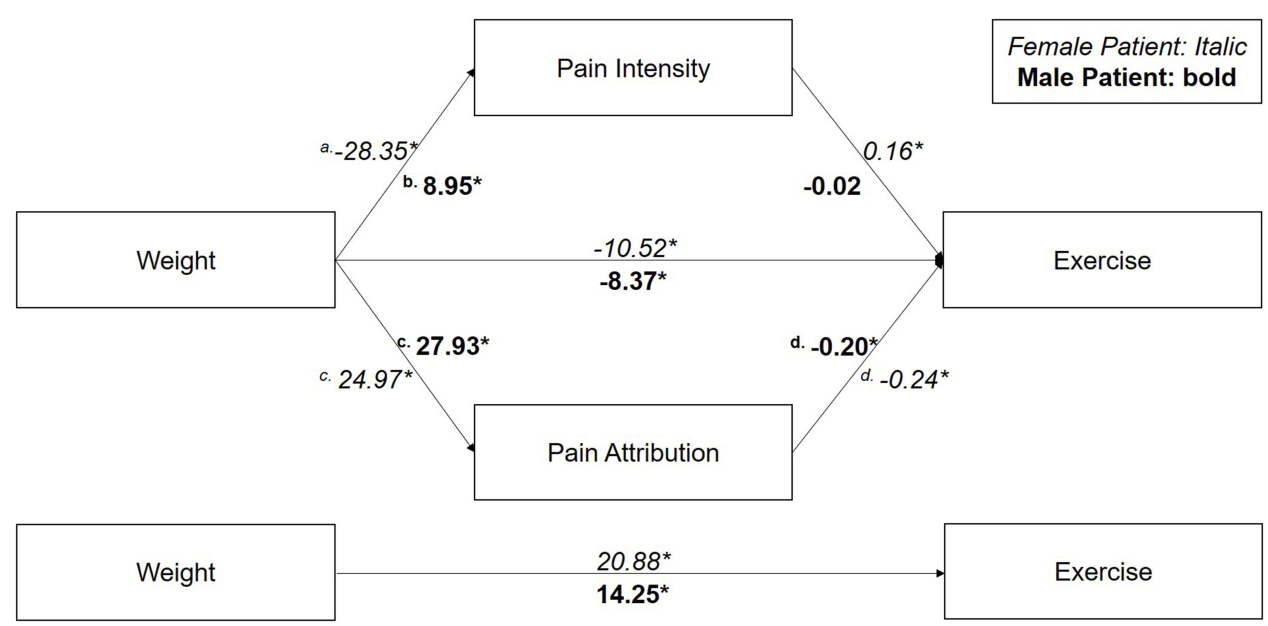

Figure 2 Mediation model for weight on exercise recommendation through pain intensity and attribution.

Note: ${ }^{a}$ Female patients with obesity were rated as being in more pain than female patients with normal weight, ${ }^{b}$ but the opposite effect was found for male patients. ${ }^{c}$ For both male and female patients, participants were more likely to attribute the cause of pain to lifestyle factors if the patient had obesity and to medical factors if the patient was normal weight. ${ }^{d}$ When participants attributed pain more to lifestyle than medical factors, they were more likely to recommend exercise to patients. *Indicates that the confidence intervals did not cross zero. 


\section{PT Recommendations}

For male patients, there was a significant total indirect effect of patient weight on the likelihood of receiving a PT recommendation via pain intensity and pain attribution (total indirect effect $=6.40 ; 95 \% \quad \mathrm{CI}: 4.26$, 8.69). Participants' pain intensity (indirect effect $=3.17$; 95\% CI: $1.97,4.56$ ) and pain attribution (indirect effect $=3.23 ; 95 \%$ CI: 1.03 , 5.53) ratings both significantly mediated the effect of patient weight on PT recommendations. Male patients with obesity were perceived to be in less pain and were more likely to have their pain attributed to lifestyle factors than to medical factors, and these differences were associated with a lower likelihood of receiving a PT recommendation compared to their normal weight counterparts. Pairwise contrasts indicated that the indirect paths through pain intensity and pain attribution were not significantly different (contrast $=-0.05 ; 95 \%$ CI: $-2.98,2.97$ ).

For female patients, there was a significant total indirect effect of patient weight on the likelihood of receiving a PT recommendation via pain intensity and pain attribution (total indirect effect $=-10.01 ; 95 \%$ CI: $-13.57,-6.40$ ). Participants' pain intensity ratings (indirect effect= -11.59 ; 95\% CI: $-14.57,-8.63)$, but not their pain attribution ratings (indirect effect $=1.58 ; 95 \% \mathrm{CI}$ : $-0.30,3.53$ ), significantly mediated the effect of patient weight on PT recommendations. Female patients with obesity were perceived to be in more pain than their normal weight counterparts, and this difference was associated with a higher likelihood of receiving a PT recommendation. Because one indirect effect was positive and the other was negative, the pairwise contrast cannot be used to determine if the indirect effects are significantly different; however, interocular examination suggests that the pain intensity effect was larger than the pain attribution effect. Figure 3 shows the results of the analysis in the model.

\section{Rest Recommendations}

For male patients, there was a significant total indirect effect of patient weight on the likelihood of receiving a rest recommendation via pain intensity and pain attribution (total indirect effect $=5.60 ; 95 \% \quad \mathrm{CI}: 3.35, \quad 7.88$ ). Participants' pain intensity (indirect effect $=2.38 ; 95 \% \mathrm{CI}$ : $1.30,3.51$ ) and attribution (indirect effect $=3.22 ; 95 \% \mathrm{CI}$ : $0.97,5.48)$ ratings both significantly mediated the effect of patient weight on rest recommendations. Compared to their normal weight counterparts, male patients with obesity were perceived to be in less pain and were more likely to have their pain attributed to lifestyle factors than to medical factors, and these differences were associated with a lower likelihood of receiving a rest recommendation. Pairwise contrasts indicated that the indirect paths through pain intensity and pain attribution were not significantly different (contrast $=-0.84 ; 95 \% \mathrm{CI}$ : $-3.52,1.88$ ).

For female patients, the total indirect effect of patient weight on the likelihood of receiving a rest recommendation via pain intensity and pain attribution was not significant (total indirect effect=-1.61; 95\% CI: $-5.77,2.40$ ); however, the specific indirect effects for both were

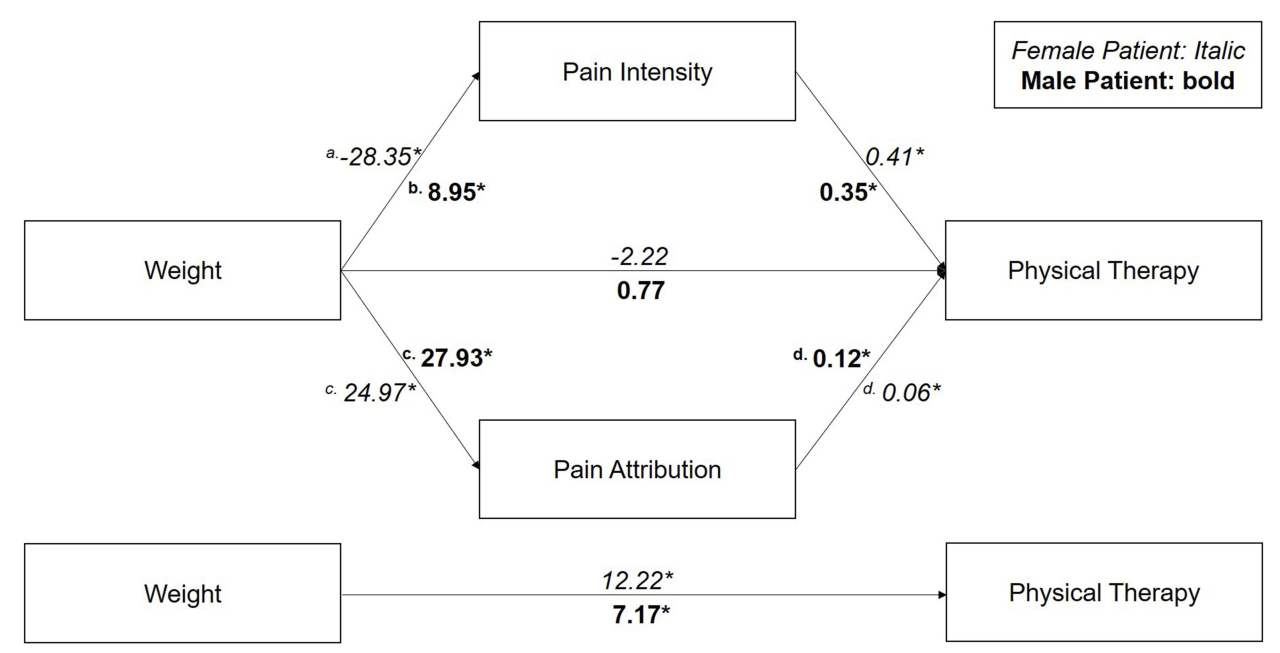

Figure 3 Mediation model for weight on physical therapy recommendation through pain intensity and attribution.

Note: ${ }^{a}$ Female patients with obesity were rated as being in more pain than female patients with normal weight, bbut the opposite effect was found for male patients. ${ }^{\mathrm{c}}$ For both male and female patients, participants were more likely to attribute the cause of the pain to lifestyle factors if the patient had obesity and to medical factors if the patient was normal weight. ${ }^{d}$ When participants attributed pain more to lifestyle than medical factors, they were less likely to recommend PT to patients. *Indicates that the confidence intervals did not cross zero. 
significant. Because the specific indirect effects were in opposite directions - negative for pain intensity (indirect effect $=-5.35$; 95\% CI: $-8.48,-2.31$ ) and positive for pain attribution (indirect effect $=3.74 ; 95 \%$ CI: 1.48, 6.08) they cancelled each other out, leading to a non-significant total indirect effect as well as a non-significant total effect for the model (total effect $=2.21 ; 95 \% \mathrm{CI}:-0.29,4.72$ ). Results of the specific effects indicated that female patients with obesity were perceived to be in more pain, which was associated with a higher likelihood of receiving a rest recommendation compared to their normal weight counterparts. Female patients with obesity also were more likely to have their pain attributed to lifestyle factors than their normal weight counterparts; however, this difference was associated with a lower likelihood of receiving a rest recommendation. Because one indirect effect was positive and the other was negative, the pairwise contrast cannot be used to determine if the indirect effects are significantly different; however, interocular examination suggests that the effects were similar in magnitude. Figure 4 shows the results of the analysis in the model.

\section{Discussion}

The current study examined the influence of patient weight and gender on laypeople's physical activity-related recommendations for chronic pain. We also examined the mediating role that perceived pain intensity and pain attribution played in the above relationship. Consistent with hypothesis one, weight and gender interacted to significantly influence exercise, PT, and rest recommendations. Hypothesis two was also supported, such that pain intensity and pain attribution mediated the relationship between weight and activity recommendations. Moreover, these mediation relationships differed across gender and activity recommendations.

Male and female patients with obesity were more likely to receive an exercise recommendation than normal weight patients. The pattern of findings was different for PT. Normal weight male patients were more likely to receive a $\mathrm{PT}$ recommendation than male patients with obesity, but the pattern was reversed for women (normal weight women were less likely to receive a PT recommendation than women with obesity). For both men and women, normal weight patients were more likely to receive a rest recommendation than were patients with obesity, and this difference was more pronounced for male patients. These weight-by-gender interactions are consistent with previous findings that patient characteristics, specifically demographic features like race and gender, influence providers' opinions and recommendations for pain management. ${ }^{10,14,15}$ Similar to medical staff and other providers included in previous studies, ${ }^{9,15}$ the laypeople in our study gave different rest recommendations according to the weight and gender of the patient. This is important given that current pain treatment guidelines encourage activity and discourage bed rest. ${ }^{2,38}$

We additionally explored participant-rated pain intensity and pain attribution as reasons why these activity-related

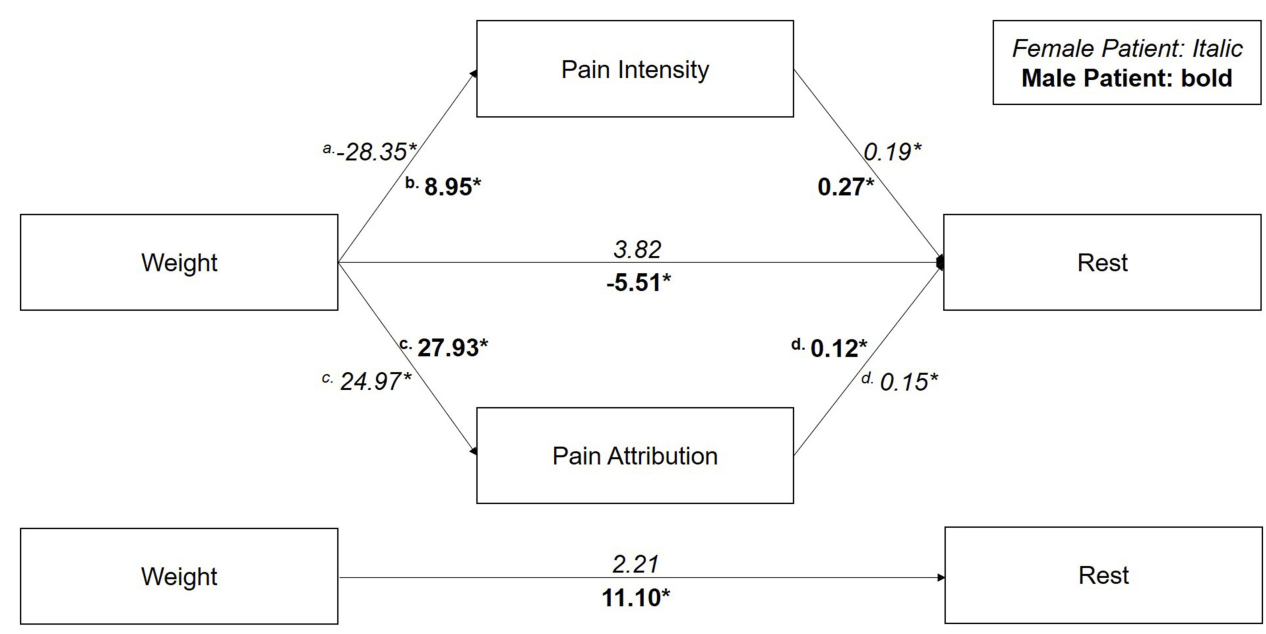

Figure 4 Mediation model for weight on rest recommendation through pain intensity and attribution.

Note: ${ }^{a}$ Female patients with obesity were rated as being in more pain than female patients with normal weight, ${ }^{b}$ but the opposite effect was found for male patients. ${ }^{c}$ For both male and female patients, participants were more likely to attribute the cause of pain to lifestyle factors if the patient had obesity and to medical factors if the patient

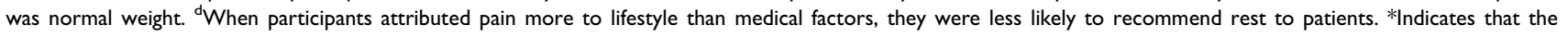
confidence intervals did not cross zero. 
recommendations varied across weight and gender. The results indicated that both factors mediated the relationship between patient weight and activity recommendations, however, these effects differed for male and female patients. For female patients, higher pain intensity ratings were associated with higher recommendations for exercise, PT, and rest. For male patients, having a lower pain intensity rating was associated with a higher likelihood of receiving recommendations for exercise, but a lower likelihood of receiving recommendations for PT and rest. Compared to normal weight counterparts, both male and female patients with obesity had the cause of their pain attributed more to lifestyle than medical factors - this difference was associated with a higher likelihood of receiving an exercise recommendation and a lower likelihood of receiving PT or rest recommendations. To our knowledge, this is the first study to use experimental methods to examine how perceptions of pain intensity and attribution influence physical activity recommendations. Our findings support previous findings that suggest symptom severity influences such recommendations. ${ }^{9,39}$ Further, our findings indicate that the influence of pain intensity differs depending on patient gender and type of activity recommendation and likewise align with healthcare providers' self-report that pain history, including cause of pain, influences their recommendations. ${ }^{39}$

Perceptions of pain intensity and pain attribution in others may be informed by two competing frameworks biomedical or biopsychosocial. A fundamental tenet of the biomedical model is that illness has a biological cause. ${ }^{40,41}$ In this sense, pain is assumed to have medical (ie, biological) underpinnings. Alternatively, observers - whether laypersons or providers - may assume a more biopsychosocial perspective, which holds that biological, psychological, and social factors interact to affect illness and health. $^{40,41}$ From this perspective, pain may be informed by a number of causal/contributing factors owing less to medical pathology (eg, tissue damage) and more to lifestyle factors (eg, behaviors) and psychosocial influences (eg, maladaptive cognitions). Although observers from either framework may attribute pain to medical or lifestyle factors, those from the biomedical perspective who attribute pain to a medical cause may be reluctant to make activity-related recommendations given that such activities may be seen as failing to address the underlying biological cause of pain. However, if individuals from a biomedical perspective consider the pain to be caused by lifestyle or psychological factors, they could be more willing to recommend lifestyle adjustments such as exercise and dietary changes. While the biopsychosocial model is the standard of care, especially for chronic pain, ${ }^{42}$ evidence suggests that many medical schools fail to educate students on the psychosocial aspects of pain ${ }^{43-45}$ thus, the biomedical model continues to dominate clinical practice.

While obesity has been found to increase pain through biological mechanisms such as inflammation or pressure on the joints, ${ }^{46}$ the cause of obesity is often seen as a personal failure attributed to lifestyle factors. ${ }^{21,47,48}$ Research also suggests that women are more likely than men to have their pain attributed to psychological factors. ${ }^{15,33,49}$ Collectively, the aforementioned research may help explain the current findings that both male and female patients with obesity - but particularly female patients - were more likely to have their pain attributed to lifestyle causes and, consequently, were more likely to be recommended exercise than normal weight patients. This interpretation may be particularly relevant for participants grounded in a biomedical perspective. In contrast, individuals with a biopsychosocial perspective may be more likely to view physical activity as a legitimate treatment that can improve pain regardless of its source. Consequently, they may be more likely to recommend physical activity for pain regardless of patients' weight, gender, or underlying cause. Given that the biomedical perspective is common among laypeople and providers in the USA, ${ }^{4,50}$ this could be a potential barrier to physical activity recommendations for patients with characteristics that lead a provider to believe that there is a medical cause of the pain. A different pattern was found for PT, suggesting that PT may be viewed differently than exercise as an intervention. While we could not statistically compare the male and female mediation models, it appears that participant rated pain intensity had a larger absolute and relative (to attribution) indirect (ie, mediated) impact on PT recommendations for female patients, whereas participant rated pain attribution had a larger absolute (but not relative) indirect impact on PT recommendations for male patients. It is likely that participants viewed pain intensity and attribution as differentially important when recommending PT or exercise depending on gender of the patient.

Fear-avoidance beliefs may also play a role in the relationships observed herein. Observers (and patients) with such beliefs are overly concerned about the potential pain- and injury-inducing effects of physical activity. ${ }^{51,52}$ Previous research has demonstrated that when providers score high on fear-avoidance measures, they are more likely to recommend bed rest and less likely to recommend physical activity for patients with back pain. ${ }^{53}$ Observers' fear-avoidance beliefs may have differential effects for 
patients of varying weights. For example, if pain is attributed to lifestyle factors (as tended to be the case for patients with obesity), observers may be less concerned (fearful) about recommending exercise. In contrast, if pain is attributed to a medical cause or injury (as tended to be the case for normal weight patients), observers may have higher fear about exacerbating this underlying biological cause, which may elicit recommendations for rest instead of activity. Similarly, previous studies have found that women are more likely to have their pain attributed to psychological factors; ${ }^{15,28,35}$ as such, providers may be less fearful about recommending they engage in physical activity (rather than rest) compared to men whose pain is more often attributed to medical factors or injury. Although these weight and gender "main effects" have a simple and intuitive appeal, the current results suggest a more complex interaction between patient weight and gender on observers' pain attributions and, consequently, physical activity recommendations. Future research is needed to determine whether and how observer fear and conceptual orientation to pain fits into these relationships.

\section{Strengths And Limitations}

Previous research has been limited by the use of nonexperimental methods like chart reviews or the use of clinical vignettes that fail to reflect a clinical setting. Our study involved videos of real patients that are standardized and used an experimental design which allows for greater confidence in causal inferences. Moreover, participants were from two different regions of the country, which increases the generalizability of the results. However, because participants were laypersons, it is an open question as to whether these results generalize to health care providers. Additionally, the high female to male participant ratio may limit generalizability given evidence that male and female providers differ in their analgesic prescribing decisions. ${ }^{54}$ Further, our results are limited by the relatively young age of participants. Much like pain sensitivity changes over time, ${ }^{55}$ treatment recommendations to others may be influenced by one's own experiences of pain. Therefore, it would be worthwhile to investigate these relationships in an older sample of participants. Our study is also limited by the use of videos of all White, middle-aged patients. Given previous findings about the impact of both race and age on provider decisions, ${ }^{56,57}$ future studies may explore these effects across different race and age groups. As the study was completed online, we were unable to account for environmental factors, such as the presence of other people that may have influenced participants' recommendations. Finally, potentially important information about participant characteristics, such as weight/BMI, fear-avoidance beliefs, and knowledge/experience concerning pain treatments, were not collected but should be explored in future studies.

\section{Implications}

The current findings suggest that laypeople hold weight and gender-related beliefs that impact their opinion on physical activity recommendations for patients in pain. By examining these relationships in laypeople, we can better understand when and where these biases may develop. Given the relatively young age of the sample, our findings suggest that these biases may develop early in life, before (future) providers enter medical training programs. Because these biases may be linked to age and/or experience, future longitudinal research should examine their within- and between-person change over time. The consequences of such biases are important, given that chronic pain and obesity are highly comorbid, ${ }^{16-20}$ physical activity is a critical component of chronic pain care, ${ }^{4,58}$ patients who receive a recommendation to become physically active are more likely to adhere to physical activity guidelines, ${ }^{59}$ and many patients are hesitant or afraid to participate in physical activity without their doctors' approval. ${ }^{60,61}$ If the findings from the current study are replicated in providers, interventions may need to be developed to reduce provider biases and increase their awareness of evidence-based activity interventions for patients regardless of weight and gender.

\section{Disclosure}

The authors report no conflicts of interest in this work.

\section{References}

1. Paley CAJ. M. I. Physical activity to reduce systemic inflammation associated with chronic pain and obesity a narrative review. Clin J Pain. 2016;32(4):365-370. doi:10.1097/AJP.0000000000000258

2. Ambrose KR, Golightly YM. Physical exercise as non-pharmacological treatment of chronic pain: why and when. Best Pract Res Clin Rheumatol. 2015;29(1):120-130. doi:10.1016/j.berh.2015.04.022

3. Hayden JA, van Tulder MW, Tomlinson G. Systematic review: strategies for using exercise therapy to improve outcomes in chronic low back pain. Ann Intern Med. 2005;142(9):776-785. doi:10.7326/00034819-142-9-200505030-00014

4. Mior S. Exercise in the treatment of chronic pain. Clin J Pain. 2001;17 (4):S77-S85. doi:10.1097/00002508-200112001-00016

5. Mekary RA, Feskanich D, Malspeis S, Hu FB, Willett WC, Field AE. Physical activity patterns and prevention of weight gain in premenopausal women. Int J Obes. 2009;33(9):1039-1047. doi:10.1038/ijo.20 09.127 
6. Coakley EH, Rimm EB, Colditz G, Kawachi I, Willett W. Predictors of weight change in men: results from the health professionals follow-up study. Int J Obes. 1998;22(2):89-96. doi:10.1038/sj.ijo.080 0549

7. Fritz JM, Childs JD, Wainner RS, Flynn TW. Primary care referral of patients with low back pain to physical therapy impact on future health Care utilization and costs. Spine. 2012;37(25):2114-2121. doi:10.1097/BRS.0b013e31825d32f5

8. Freburger JK, Carey TS, Holmes GM, et al. Exercise prescription for chronic back or neck pain: who prescribes it? Who gets it? What is prescribed? Arthritis Rheum-Arthritis Care Res. 2009;61(2):192-200. doi:10.1002/art.24234

9. Rainville J, Carlson N, Polatin P, Gatchel RJ, Indahl A. Exploration of physicians' recommendations for activities in chronic low back pain. Spine. 2000;25(17):2210-2220. doi:10.1097/00007632-200009 010-00012

10. Nelson A. Unequal treatment: confronting racial and ethnic disparities in health care. J Natl Med Assoc. 2002;94(8):666-668.

11. De Ruddere L, Craig KD. Understanding stigma and chronic pain: astate-of-the-art review. Pain. 2016;157(8):1607-1610. doi:10.1097/j. pain.0000000000000512

12. De Ruddere L, Goubert L, Stevens M, Williams ACD, Crombez G. Discounting pain in the absence of medical evidence is explained by negative evaluation of the patient. Pain. 2013;154(5):669-676. doi:10.1016/j.pain.2012.12.018

13. De Ruddere L, Goubert L, Steyens MAL, Deyeugele M, Craig KD, Crombez G. Health care professionals' reactions to patient pain: impact of knowledge about medical evidence and psychosocial influences. J Pain. 2014;15(3):262-270. doi:10.1016/j.jpain.2013.11.002

14. Fontaine KR, Bartlett SJ, Heo M. Are health care professionals advising adults with arthritis to become more physically active? Arthritis Rheum. 2005;53(2):279-283. doi:10.1002/(ISSN)1529-0131

15. Miller MM, Allison A, Trost Z, et al. Differential effect of patient weight on pain-related judgements about male and female chronic low back pain patients. J Pain. 2018;19(1):57-66. doi:10.1016/j. jpain.2017.09.001

16. Bigal ME, Liberman JN, Lipton RB. Obesity and migraine - A population study. Neurology. 2006;66(4):545-550. doi:10.1212/01. wnl.0000197218.05284.82

17. Chai NC, Scher AI, Moghekar A, Bond DS, Peterlin BL. Obesity and headache: part I - A systematic review of the epidemiology of obesity and headache. Headache. 2014;54(2):219-234. doi:10.1111/head.12 296

18. Okifuji A, Donaldson GW, Barck L, Fine PG. Relationship between fibromyalgia and obesity in pain, function, mood, and sleep. J Pain. 2010;11(12):1329-1337. doi:10.1016/j.jpain.2010.03.006

19. Okifuji A, Hare BD. The association between chronic pain and obesity. J Pain Res. 2015;8:399-408. doi:10.2147/JPR.S55598

20. Shiri R, Karppinen J, Leino-Arjas P, Solovieva S, Viikari-Juntura E. The association between obesity and low back pain: a meta-analysis. Am J Epidemiol. 2010;171(2):135-154. doi:10.1093/aje/kwp356

21. Puhl RM, Heuer CA. The stigma of obesity: a review and update. Obesity. 2009;17(5):941-964. doi:10.1038/oby.2008.636

22. Schvey NA, Sbrocco T, Bakalar JL, et al. The experience of weight stigma among gym members with overweight and obesity. Stigma Health. 2017;2(4):292-306. doi:10.1037/sah0000062

23. Mensinger JL, Meadows A. Internalized weight stigma mediates and moderates physical activity outcomes during a healthy living program for women with high body mass index. Psychol Sport Exerc. 2017;30:64-72. doi:10.1016/j.psychsport.2017.01.010

24. Robertson N, Vohora R. Fitness vs. fatness: implicit bias towards obesity among fitness professionals and regular exercisers. Psychol Sport Exerc. 2008;9(4):547-557. doi:10.1016/j.psychsport.2007.06.002

25. Fikkan JL, Rothblum ED. Is fat a feminist issue? Exploring the gendered nature of weight bias. Sex Roles. 2012;66(9-10):575-592. doi:10.1007/s11199-011-0022-5
26. Phelan SM, Burgess DJ, Yeazel MW, Hellerstedt WL, Griffin JM, Ryn VM. Impact of weight bias and stigma on quality of care and outcomes for patients with obesity. Obesity Rev. 2015;16(4):319-326. doi:10.1111/obr.2015.16.issue-4

27. Chalabaev A, Sarrazin P, Fontayne P, Boiché J, Clément-Guillotin C. The influence of sex stereotypes and gender roles on participation and performance in sport and exercise: review and future directions. Psychol Sport Exerc. 2013;14(2):136-144. doi:10.1016/j.psychsport. 2012.10.005

28. Knisel E, Opitz S, Wossmann M, Keteihuf K. Sport motivation and physical activity of students in three European schools. Int $J$ Phys Educ. 2009;46(2):40-53.

29. Hoffmann DE, Tarzian AJ. The girl who cried pain: a bias against women in the treatment of pain. J Law Med Ethics. 2001;28 (4_suppl):13-27.

30. Institute Of Medicine. Relieving Pain In America: A Blueprint For Transforming Prevention, Care, Education, And Research. Washington, DC: National Academies Press; 2011.

31. Wandner LD, Heft MW, Lok BC, et al. The impact of patients' gender, race, and age on health care professionals' pain management decisions: an online survey using virtual human technology. Int $J$ Nurs Stud. 2014;51(5):726-733. doi:10.1016/j.ijnurstu.2013.09.011

32. Pierik JG, IJzerman MJ, Gaakeer MI, Vollenbroek-Hutten MM, Doggen CJ. Painful discrimination in the emergency department: risk factors for underassessment of patients' pain by nurses. $J$ Emergency Nurs. 2017;43(3):228-238. doi:10.1016/j.jen.2016.10. 007

33. Hirsh AT, Hollingshead NA, Bair MJ, Matthias MS, Wu J, Kroenke $\mathrm{K}$. The influence of patient's sex, race and depression on clinician pain treatment decisions. Eur J Pain. 2013;17(10):1569-1579.

34. Torres CA, Bartley EJ, Wandner LD, Alqudah AF, Hirsh AT, Robinson ME. The influence of sex, race, and age on pain assessment and treatment decisions using virtual human technology: a crossnational comparison. $J$ Pain Res. 2013;6:577-588.

35. Bartley EJ, Fillingim RB. Sex differences in pain: a brief review of clinical and experimental findings. Br J Anaesth. 2013;111(1):52-58. doi:10.1093/bja/aet127

36. Division of Nutrition PA, and Obesity, National Center for Chronic Disease Prevention and Health Promotion. Defining adult overweight and obesity; 2017. Avaialble from: https://www.cdc.gov/obesity/ adult/defining.html. Accessed September 06, 2019.

37. Tabachnick BG, Fidell LS. Using Multivariate Statistics. 6th ed. Boston, MA: Pearson; 2013.

38. Services USDoHaH. Pain management best practices inter-agency task force report: updates, gaps, inconsistencies, and recommendations; 2019. Avaialble from: https://www.hhs.gov/ash/advisory-com mittees/pain/reports/index.html. Accessed September 06, 2019.

39. Hollingshead NA, Meints S, Middleton SK, Free CA, Hirsh AT. Examining influential factors in providers' chronic pain treatment decisions: a comparison of physicians and medical students. $B M C$ Med Educ. 2015;15:164. doi:10.1186/s12909-015-0441-z

40. Straub RO. Health Psychology: A Biopsychosocial Approach. Macmillan; 2006.

41. Engel GL. The need for a new medical model: a challenge for biomedicine. Science. 1977;196(4286):129-136. doi:10.1126/science. 847460

42. Kamper SJ, Apeldoorn A, Chiarotto A, et al. Multidisciplinary biopsychosocial rehabilitation for chronic low back pain: cochrane systematic review and meta-analysis. $B M J .2015 ; 350: \mathrm{h} 444$.

43. Jaini PA, Lee JS-H. A review of 21 st century utility of a biopsychosocial model in United States medical school education. $J$ Lifestyle Med. 2015;5(2):49-59. doi:10.15280/jlm.2015.5.2.49

44. Astin JA, Sierpina VS, Forys K, Clarridge B. Integration of the biopsychosocial model: perspectives of medical students and residents. Acad Med. 2008;83(1):20-27. doi:10.1097/ACM.0b013e3181 $5 \mathrm{c} 61 \mathrm{~b} 0$ 
45. Williams BC, Ward DA, Chick DA, Johnson EL, Ross PT. Using a six-domain framework to include biopsychosocial information in the standard medical history. Teach Learn Med. 2019;31(1):87-98. doi:10.1080/10401334.2018.1480958

46. McVinnie DS. Obesity and pain. Br J Pain. 2013;7(4):163-170. doi:10.1177/2049463713484296

47. Throsby K. "How could you let yourself get like that?": stories of the origins of obesity in accounts of weight loss surgery. Soc Sci Med. 2007;65(8):1561-1571. doi:10.1016/j.socscimed.2007.06.005

48. Puhl R, Brownell KD. Bias, discrimination, and obesity. Obes Res. 2001;9(12):788-805. doi:10.1038/oby.2001.108

49. Hirsh AT, Hollingshead NA, Matthias MS, Bair MJ, Kroenke K. The influence of patient sex, provider sex, and sexist attitudes on pain treatment decisions. J Pain. 2014;15(5):551-559. doi:10.1016/j. jpain.2014.02.003

50. Astin JA, Goddard TG, Forys K. Barriers to the integration of mind-body medicine: perceptions of physicians, residents, and medical students. Explore. 2005;1(4):278-283. doi:10.1016/j. explore.2005.04.014

51. Houben R, Gijsen A, Peterson J, De Jong P, Vlaeyen J. Do health care providers' attitudes towards back pain predict their treatment recommendations? Differential predictive validity of implicit and explicit attitude measures. Pain. 2005;114(3):491-498. doi:10.1016/ j.pain.2005.01.017

52. Rainville J, Bagnall D, Phalen L. Health care providers' attitudes and beliefs about functional impairments and chronic back pain. Clin J Pain. 1995;11(4):287-295. doi:10.1097/00002508-199512000-00006

53. Coudeyre E, Rannou F, Tubach F, et al. General practitioners' fearavoidance beliefs influence their management of patients with low back pain. Pain. 2006;124(3):330-337. doi:10.1016/j.pain.2006.05.003
54. Weisse CS, Sorum PC, Sanders KN, Syat BL. Do gender and race affect decisions about pain management? J Gen Intern Med. 2001;16 (4):211-217. doi:10.1046/j.1525-1497.2001.016004211.x

55. El Tumi H, Johnson M, Dantas P, Maynard M, Tashani O. Agerelated changes in pain sensitivity in healthy humans: a systematic review with meta-analysis. Eur J Pain. 2017;21(6):955-964. doi:10. 1002/ejp.2017.21.issue-6

56. Green CR, Anderson KO, Baker TA, et al. The unequal burden of pain: confronting racial and ethnic disparities in pain. Pain Med. 2003;4(3):277-294. doi:10.1046/j.1526-4637.2003.03034.x

57. Austin S, Qu H, Shewchuk RM. Age bias in physicians' recommendations for physical activity: a behavioral model of healthcare utilization for adults with arthritis. J Phys Act Health. 2013;10(2):222-231. doi:10.1123/jpah.10.2.222

58. Geneen LJ, Moore RA, Clarke C, Martin D, Colvin LA, Smith BH. Physical activity and exercise for chronic pain in adults: an overview of Cochrane Reviews. Cochrane Database of Systematic Reviews. 2017, Issue 4: Article No: CD011279.

59. Austin S, Qu H, Shewchuk RM. Health Care Providers' Recommendations for Physical Activity and Adherence to Physical Activity Guidelines Among Adults With Arthritis. Prev Chronic Dis. 2013;10:130077.

60. Stenberg G, Fjellman-Wiklund A, Ahlgren C. 'I am afraid to make the damage worse'-fear of engaging in physical activity among patients with neck or back pain-a gender perspective. Scand J Caring Sci. 2014;28(1):146-154. doi:10.1111/scs.2014.28.issue-1

61. Alschuler KN, Hoodin F, Murphy SL, Rice J, Geisser ME. Factors contributing to physical activity in a chronic low back pain clinical sample: a comprehensive analysis using continuous ambulatory monitoring. Pain. 2011;152(11):2521-2527. doi:10.1016/j.pain.2011.07.017
Journal of Pain Research

\section{Publish your work in this journal}

The Journal of Pain Research is an international, peer reviewed, open access, online journal that welcomes laboratory and clinical findings in the fields of pain research and the prevention and management of pain. Original research, reviews, symposium reports, hypothesis formation and commentaries are all considered for publication. The manuscript management system is completely online and includes a very quick and fair peer-review system, which is all easy to use. Visit http:// www.dovepress.com/testimonials.php to read real quotes from published authors. 